\title{
Research of a Precise Road Load Test Method
}

\author{
Xu Jun Hui ${ }^{1, a}$, Zhang Peng ${ }^{2, b}$ and Xu Dandan ${ }^{3, c}$ \\ ${ }_{1,2,3}$ No. 68,East Xianfeng Road, Dongli District, Tianjin, China \\ axujunhui@catarc.ac.cn, bzhangpeng@catarc.ac.cn, xudandan@catarc.ac.cn
}

Keywords: vehicle, road load, onboard anemometry, accuracy.

Abstract. At present, the measurement of vehicle road load, in other word running resistance, are mostly coast down methods. Due to the influence of wind, the method has the problems of poor repeatability and accuracy. This paper, according the current domestic and foreign automobile road load measurement regulations and test methods, introduced a precise measurement of the vehicle road load. By installing a vehicle-mounted anemometer, we measure the absolute speed of the car and the relative speed of the car to the wind at the same time, as the car coast down in the neutral road, and then calculates the car's resistance at each speed point, which is, road load. Through theoretical analysis and a number of test results show that the consistency of the test results obtained by the method significantly improved, by modifying the impact of wind speed, the test results can better characterize the vehicle's road load.

\section{Introduction}

Under the requirements of low energy consumption and environmental protection, automotive energy-saving and emission testing requirements are getting higher and higher. There are many certification tests and research and development tests that need to be completed on a chassis dynamometer. How to accurately measure the road load, which means running resistance, of a vehicle and then simulate the resistance on dynamometer is a very important research area for domestic and foreign research institutions. At present, there are mainly three kinds of existing test methods: road coast down energy change method, torque method, wind tunnel and chassis dynamometer test combination method [1]. Among them, the road coast down method is the most widely used method because it only needs the speed measuring instrument such as GPS to be easy to install and implement. However, affected by wind speed and road slop, the accuracy and consistency are very poor. The torque method needs to fix strain gauges on the driving axle shaft of the vehicle. And the test cycle by using this method is too long, and it is also easy to interference by the wind and slop. For the combined wind tunnel and dynamometer tests, the wind tunnel laboratory is used to measure the drag coefficient, and then the dynamometer is used to measure the mechanical resistance inside the vehicle and tire resistance together. The test method is carried out in the laboratory. Therefore, various conditions are easy to control and the accuracy is also considerably high. However, the resources of the wind tunnel laboratory are scarce, and thus it is not an effective measure to measure the running resistance.

Table 1: Comparison of test methods

\begin{tabular}{cccc}
\hline Test method & Complexity & Testing costs & Accuracy \\
\hline Coast down method & Low & Low & Low \\
Torque method & Medium & Medium & Low \\
Wind tunnel and dyno method & High & High & High \\
\hline
\end{tabular}

The coast down method is simple, and it is limited by the effects of wind speed. The accuracy of measurement results can be improved by using an anemometer fixed on the vehicle. So that we can measure absolute speed of the vehicle by GPS and speed of vehicle relative to air and correct the influence of the wind speed simultaneously. The accuracy of the measurement results significantly improved and the consistency of the results of multiple tests also ensured. 


\section{The expression form of road load}

Normally, the quadratic equation that simulates the driving resistance of the vehicle on the chassis dynamometer is the one-dimensional quadratic equation of the vehicle's driving speed.

$$
\mathrm{F}=\mathrm{A}+\mathrm{Bv}+\mathrm{Cv}^{2}
$$

Where $\mathrm{F}$ is the vehicle's resistance at each vehicle speed, $\mathrm{v}$ is the vehicle's driving speed, and $\mathrm{A}, \mathrm{B}$, and $\mathrm{C}$ are constant speed coefficient, one-term coefficient and quadratic coefficient, respectively. A, $\mathrm{B}$ and $\mathrm{C}$ determined by the road coast down test method are the target road load coefficients. This coefficient is the target value of the chassis dynamometer. The actual resistance and target resistance value of the chassis dynamometer are determined by adjusting the parameters of the chassis dynamometer combined with the actual resistance of the vehicle under the state of the stock transfer as close as possible to the target resistance value. When we use the actual coefficient, we allow $2 \%-3 \%$ deviation usually [2].

According to Newton's theory and automobile theory, the equation of motion of a vehicle is

$$
\mathrm{m}_{\mathrm{e}}(\mathrm{dv} / \mathrm{dt})=\mathrm{D}_{\text {mech }}+\mathrm{D}_{\text {aero }}+\mathrm{D}_{\text {grav }}
$$

Where $m_{e}$ represents the effective quality of the car, $D_{\text {mech }}$ represents the mechanical resistance of the car. $D_{\text {areo, }}$, represents the air resistance of the vehicle, $D_{\text {grav }}$ represents the resistance of slop $[3,4]$. The above three aspects of resistance analysis: (i) Mechanical resistance from the transmission system, tires, etc., is generally a quadratic function of speed. (ii) The aerodynamic resistance is closely related to the exterior shape of the vehicle, such as the frontal area. Because the wind speed and the direction of travel of the vehicle have a vector angle, the aerodynamic drag is proportional to the windward surface of the vehicle, air density, the square of apparent vehicle speed. (iii) Under normal circumstances, the vertical slope of the test road surface is not more than $0.1 \%$ in the test field for vehicle certification in China, so this item can be ignored in the actual calculation. After the hypothesis and theoretical deduction, the final equation of motion is

$$
m_{e}(d v / d t)=A_{m}+B_{m} v+C_{m} v^{2}+1 / 2 \rho A_{f} v_{r}^{2}\left(a_{0}+a_{1} Y+a_{2} Y^{2}+a_{3} Y^{3}+a_{4} Y^{4}\right)
$$

Where $A_{m}, B_{m}$ and $C_{m}$ are constant terms that characterizes the mechanical resistance and tire resistance of a vehicle, $\mathrm{a}_{0}, \mathrm{a}_{1}, \mathrm{a}_{2}, \mathrm{a}_{3}, \mathrm{a}_{4}$, represent the constant terms that characterizes aerodynamic parameters and $A_{f}$ is the frontal area of the vehicle. When the frontal area is known, constrained analysis can be used to decompose the road load into aerodynamics resistance and mechanical resistance. When the frontal area is unknown, a numerical calculation method can be used to analyze the integrated road load factors $[5,6]$.

\section{Road load test using onboard anemometry method}

Based on the Eq. 3 of motion function, the equipment required for designing the test includes: (i) GPS for measuring vehicle speed v. (ii) Vehicle anemometer for measuring relative wind speed $v_{r}$. (iii) Encoder for measuring the angle of wind relative to vehicle speed, which means yaw angle Y. (iv) Atmospheric temperature, using a thermocouple sensor with high accuracy and high speed.

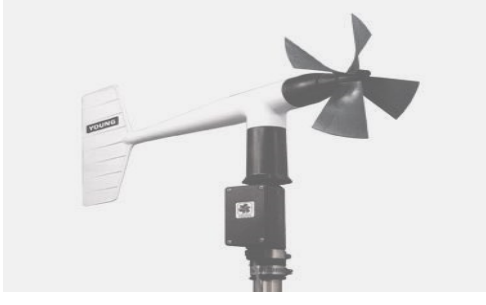

Fig 1: Anemometer

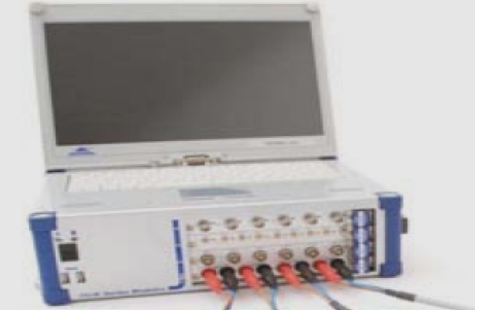

Fig 2: Data acquisition system 
The selected vehicle anemometer can measure wind speed and rotation angle and the measurement signal passes by a data acquisition device. In addition, this test method requires that the vehicle anemometer be installed in a location that has the least impact on the vehicle's operating performance. From an aerodynamic analysis, the anemometer should be installed $2 \mathrm{~m}$ in front of the vehicle's station point. At the same time, the anemometer should be installed parallel to the road surface. Leveling can be done on the anemoscope bracket.

Select vehicle 1\# category of M2 with 15 seats and vehicle 2\# category of M1 with 5 seats for road load measurement tests.

Table 2: Test vehicles

\begin{tabular}{ccc}
\hline Item & $01 \#$ & $02 \#$ \\
\hline Test mess $[\mathrm{kg}]$ & 2430 & 1430 \\
Length, width, height $[\mathrm{mm}]$ & $5380 \times 1920 \times 2285$ & $5380 \times 1920 \times 2285$ \\
Tire specifications & $195 \mathrm{R} 15 \mathrm{LT}, 350[\mathrm{kPa}]$ & $215 / 65 \mathrm{R} 16,230[\mathrm{kPa}]$ \\
Engine model & $3 \mathrm{TZ}$ & $\mathrm{M} 20 \mathrm{~A}$ \\
Transmission model & ZM025 & K120 \\
Main reduction ratio & 4.875 & 4.014
\end{tabular}

The vehicle $1 \#$ accelerates to $125 \mathrm{~km} / \mathrm{h}$. After the shift lever is taken to the neutral position, the throttle is released and the taxi speed is $15 \mathrm{~km} / \mathrm{h}$. The vehicle $2 \#$ speeds up to $145 \mathrm{~km} / \mathrm{h}$. After the gear shift to neutral, the throttle is released and the taxi speed is $15 \mathrm{~km} / \mathrm{h}$. Each selected vehicle carries out at least five complete round trips. In order to ensure data validity, the sampling frequency of the data acquisition system is at least $5 \mathrm{~Hz}$. After the original data is collected, the data is filtered to $1 \mathrm{~Hz}$.

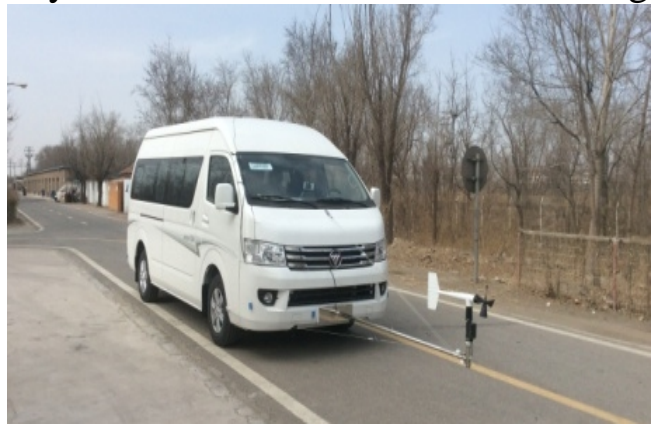

Fig 3: test vehicle category of M2

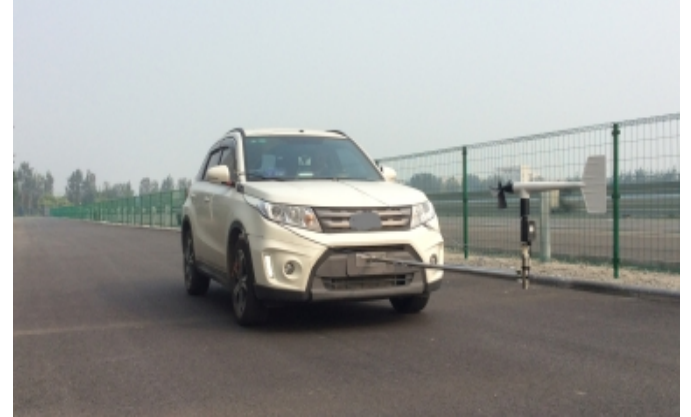

Fig 4: test vehicle category of M1

First, the invalid data points in the original gliding test data are eliminated, data points whose angle between wind speed and wind speed exceeding 20 degrees are excluded, and data points with relative wind speed less than $5 \mathrm{~km} / \mathrm{h}$ are excluded. Then data filtering was performed. Based on the valid coast down test data, a linear regression method was used to perform a preliminary analysis of the parameters in the equation of motion. The results are shown in the table below.

Table 3: Preliminary data analysis

\begin{tabular}{ccc}
\hline Parameters & $01 \#$ & $02 \#$ \\
\hline $\mathrm{A}_{\mathrm{m}}$ & 255.846539 & 167.911 \\
$\mathrm{~B}_{\mathrm{m}}$ & 2.553220 & 0.59527 \\
$\mathrm{C}_{\mathrm{m}}$ & 0.003819 & 0.0002081 \\
$\mathrm{a}_{0}$ & 0.045569 & 0.025738 \\
$\mathrm{a}_{1}$ & -0.000569 & 0.0000136 \\
$\mathrm{a}_{2}$ & 0.000028 & 0.00008073 \\
$\mathrm{a}_{3}$ & 0.000000 & -0.0000188 \\
$\mathrm{a}_{4}$ & 0.000000 & -0.00000228
\end{tabular}

According to the preliminary analysis, the resistance of the prototype vehicle at each speed is calculated from the second to second, and compared with the predicted resistance at that point, the data points with large deviations are proposed, and after removing all the deviation points, the part of the data is finally valid. We chose those final valid data for final analysis and calculation. The linear 
regression method was used to analyze the parameters in the equation of motion. The results are shown in the following table.

Table 4: Final data analysis

\begin{tabular}{ccc}
\hline Parameters & $01 \#$ & 02\# \\
\hline $\mathrm{A}_{\mathrm{m}}$ & 288.217313 & 167.726 \\
$\mathrm{~B}_{\mathrm{m}}$ & 1.426728 & 0.60575 \\
$\mathrm{C}_{\mathrm{m}}$ & 0.010238 & 0.0003219 \\
$\mathrm{a}_{0}$ & 0.045665 & 0.0256225 \\
$\mathrm{a}_{1}$ & -0.000746 & 0.0000013 \\
$\mathrm{a}_{2}$ & 0.000052 & 0.0000668347 \\
$\mathrm{a}_{3}$ & 0.000002 & -0.0000167189 \\
$\mathrm{a}_{4}$ & 0.000000 & -0.0000019910 \\
\hline
\end{tabular}

According to the final parameter results, the test data is corrected to the standard environmental condition to obtain the target load factor.

Table 5: Target road load factor

\begin{tabular}{ccc}
\hline Road load factor & $01 \#$ & $02 \#$ \\
\hline $\mathrm{A}_{\mathrm{t}}[\mathrm{N}]$ & 262.0 & 151.0 \\
$\mathrm{~B}_{\mathrm{t}}[\mathrm{N} /[\mathrm{km} . \mathrm{h}]]$ & 1.2972 & 0.5448 \\
$\mathrm{C}_{\mathrm{t}}\left[\mathrm{N} /[\mathrm{km} . \mathrm{h}]^{\wedge} 2\right]$ & 0.0707 & 0.0345 \\
\hline
\end{tabular}

According to the frontal area of the vehicle and the density of air, the resistance of the vehicle at each speed can be divided into two parts. The first part is the mechanical resistance, and the resistance of the part is smaller. The other part is the aerodynamic resistance. The coefficient of the secondary resistance of this part is larger. When the vehicle speed increases, the resistance rises obviously. The resistance of the final synthesis is as shown in the enclosed area.

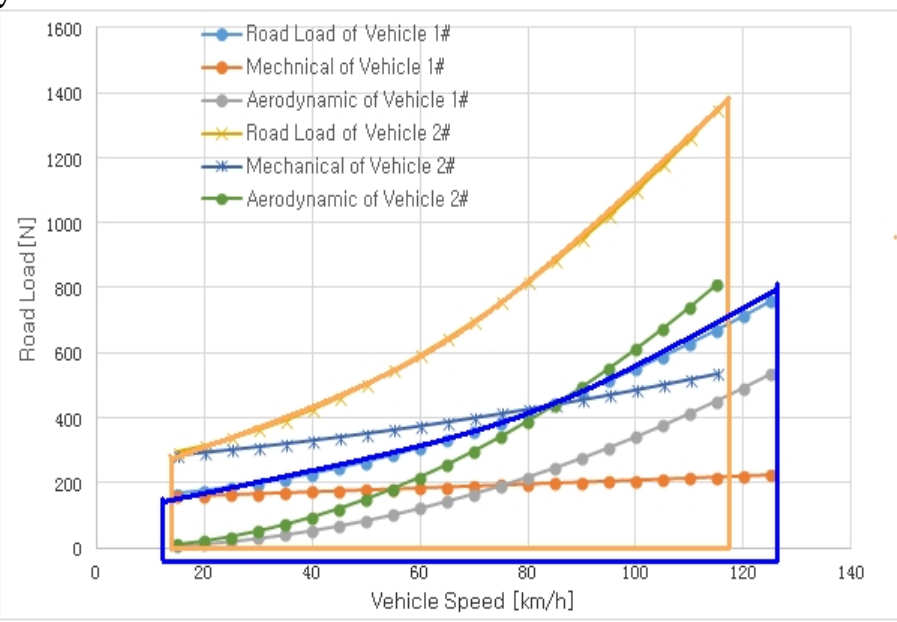

Fig5: Resistance area of vehicle 1\# and 2\#

Based on the frontal area of the vehicle and the air density, the resistance of the prototype vehicle at each speed can be divided into two parts. The first part is the mechanical resistance, shown by the red curve in the figure below. The other part is the aerodynamic resistance, as shown by the green curve. The resistance of the final synthesis is shown by the blue curve.

\section{Conclusions}

(1) Using linear regression method, first analyze all available gliding test data to obtain preliminary test results. Then comparing the observed values with the theoretical values, remove the points with large differences and use the effective data points for the final linearity. This method can effectively eliminate the accidental data during actual testing, such as gusts, road bumps, GPS interference, and other random fluctuation data. 
(2) Vehicle-mounted anemometer can minimize the impact of environmental wind speed, ensure the consistency of test data, and effectively improve the accuracy and effectiveness of the test.

(3) According to the equations of motion, a constrained analysis method can be used to separate the aerodynamic resistance from the mechanical resistance, and to better analyze the source of resistance of the tested vehicle.

(4) Convergence analysis, due to the use of numerical preliminary analysis, and then using observation correction, the randomness data is removed, the convergence is good, and the experiment can reach rapid convergence after fewer number of coast down tests.

(5) Based on the effective use of coast down test data, the test method can effectively, accurately and quickly measure the road load of the vehicle. Through comparison with the traditional data analysis results, it is found that the method can better reflect the actual road load performance.

\section{Acknowledgements}

This work was financially supported by the research project "The Research of Vehicle Road Test Evaluation", which is the major special Five-year automotive test platform of CATARC.

\section{References}

[1] Lin Xudong, Zhong Zijin, Lin Huomu, Zhang Chongwen. Reproducibility of Vehicle Resistance on Vehicle Chassis Dynamometer. Guangdong Highway Communications, 2003, Vol. 2, No. 2, 64-66.

[2] Zeng Haipeng, Hu Zhentao, Zhang Yajun, Yu Hai. Vehicle roadway gliding and its data processing. Automotive Technology, Vol.39, No.3, 36-41.

[3] SAE J2263 Vehicle Anemometer Glide Measurement Road Load Technical Document.

[4] GB 18352.6-2017 Limits and Measurement Methods for Pollutant Emission of Light Vehicles (China Sixth Stage).

[5] Liu Fucai, Pan Jianghua, Han Zongqi. Method for Measuring Vehicle Resistance Coefficient Based on Genetic Algorithm[J]. Automobile Engineering, 2003, 25(6), 610-616.

[6] Li Xiaozhao, Zhao Kegang, Huang Xiangdong, Wei Jianjun. Determination of Vehicle Resistance Model Parameters [J]. Automotive Engineering, 2011, 33(8), 645-648. 